bronchodilator (LABD) prior to randomisation affected the lungfunction benefits of $\mathrm{T}+\mathrm{O} 5 / 5 \mu \mathrm{g}$ (via Respimat ${ }^{\circledR}$ ) compared to T $5 \mu \mathrm{g}$ (via Respimat ${ }^{\circledR}$ ).

Methods In the studies, 2124 patients had not received prior LABD treatment $(\mathrm{T}+\mathrm{O} \mathrm{n}=426 ; \mathrm{T} \mathrm{n}=454)$ and 3038 patients had $(\mathrm{T}+\mathrm{O} \mathrm{n}=603, \mathrm{~T} \mathrm{n}=579 ; 60.6 \%$ LAMA, 78.8\% LABA). Baseline characteristics for all patients and a sub-group with Global initiative for chronic Obstructive Lung Disease (GOLD) 2 lung-function impairment are presented in the Table 1. Forced expiratory volume in $1 \mathrm{~s}\left(\mathrm{FEV}_{1}\right)$ area under the curve from $0-3$ $\mathrm{h}\left(\mathrm{AUC}_{0-3}\right)$ response (change from baseline) and trough $\mathrm{FEV}_{1}$ response were primary end points in the studies.

Results Comparable responses for both $\mathrm{FEV}_{1} \mathrm{AUC}_{0-3}$ and trough $\mathrm{FEV}_{1}$ were observed in patients previously treated and untreated with LABD (see Table 1). The between-treatment differences (adjusted mean response [SE]; $\mathrm{mL}$ ) for no prior LABD and prior LABD treatment, respectively, were: 116 (13) and 105 (11) for $\mathrm{FEV}_{1} \mathrm{AUC}_{0-3}$, and 76 (14) and 49 (11) for trough $\mathrm{FEV}_{1}$. In the GOLD 2 subgroup, the between-treatment differences (adjusted mean response $[\mathrm{SE}] ; \mathrm{mL}$ ) for no prior $\mathrm{LABD}$ and prior LABD treatment, respectively, were: 114 (19) and 123 (17) for $\mathrm{FEV}_{1}$ $\mathrm{AUC}_{0-3}$, and 79 (20) and 61 (18) for trough $\mathrm{FEV}_{1}$.

Conclusions Our analyses demonstrate the robust lung-function efficacy of $\mathrm{T}+\mathrm{O}$, compared to $\mathrm{T}$ alone, independent of the requirement for, or prior use of, LABD. These findings suggest a benefit of combination therapy over the mono-product as a firstline maintenance treatment.

Funding Boehringer Ingelheim.

\section{P126 EFFICACY OF ACLIDINIUM BROMIDE COMPARED WITH TIOTROPIUM AND PLACEBO IN SYMPTOMATIC PATIENTS WITH MODERATE TO SEVERE CHRONIC OBSTRUCTIVE PULMONARY DISEASE (COPD): POST-HOC ANALYSIS OF A PHASE IIIB STUDY}

1) Beier, ${ }^{2} \mathrm{R}$ Mroz, ${ }^{3} \mathrm{~F}$ Chuecos, ${ }^{3} \mathrm{E}$ Garcia Gil. ${ }^{1}$ Insaf Respiratory Research Institute, Wiesbaden, Germany; ${ }^{2}$ ISPL Centrum Medyczne, Białystok, Poland; ${ }^{3}$ AstraZeneca PLC, Barcelona, Spain

10.1136/thoraxjnl-2015-207770.263
Introduction and objective Maintaining bronchodilation and symptom control throughout the day and night is an important COPD therapeutic aim. Here, we compare 24-hour lung function and symptom control in symptomatic patients with moderate to severe COPD treated with aclidinium or tiotropium, two long-acting, muscarinic antagonists.

Methods This was a post-hoc analysis of a 6-week, double-blind, Phase IIIb study comparing aclidinium $400 \mu \mathrm{g}$ BID with tiotropium bromide $18 \mu \mathrm{g}$ QD or placebo in patients with moderate to severe COPD (NCT01462929). Symptomatic patients were defined as having an EXAcerbations of Chronic pulmonary disease Tool-Respiratory Symptoms (E-RS) baseline score $\geq 10$ units. Primary endpoint: change from baseline in normalised $\mathrm{FEV}_{1}$ AUC over 24-hours post-morning dose $\left(\mathrm{AUC}_{-24 / 24 \mathrm{~h}}\right.$ ) at Week 6. Other endpoints: change from baseline in morning predose (trough) $\mathrm{FEV}_{1}$ and change from baseline in $\mathrm{FEV}_{1} \mathrm{AUC}_{0-2 /}$ $12 \mathrm{~h} ; 12--4 / 12 \mathrm{~h}$, E-RS, early-morning and night-time symptoms, and limitation of early-morning activities.

Results A total of 277/414 symptomatic patients were included; mean age was 62.1 years, $54.5 \%$ were current smokers, baseline $\mathrm{FEV}_{1} 1.41 \pm 0.48 \mathrm{~L}$. At Week 6, aclidinium $400 \mu \mathrm{g}$ BID improved $\mathrm{FEV}_{1}$ over $24 \mathrm{~h}$ from baseline vs placebo (Table 1). During the night-time period, aclidinium $400 \mu \mathrm{g}$ BID improved $\mathrm{FEV}_{1}$ from baseline vs tiotropium $18 \mu \mathrm{g}$ QD. At Week 6, improvements in trough $\mathrm{FEV}_{1}$ from baseline were observed with aclidinium vs tiotropium and placebo. Aclidinium improved ERS total score from baseline vs tiotropium and placebo. Moreover, aclidinium improved early-morning and night-time symptom severity from baseline vs tiotropium and placebo over the treatment period (see Table 1 for all results described above). Limitation of early-morning activities caused by COPD symptoms was also improved with aclidinium vs tiotropium and placebo $(\mathrm{p}<0.05)$. Tolerability has been previously reported (Beier COPD 2013) where adverse events (AEs) were similar in each arm, few anticholinergic AEs or serious AEs occurred in any group, and aclidinium was well tolerated.

Conclusions Aclidinium $400 \mu \mathrm{g}$ BID improved bronchodilation, particularly during the night-time period, as well as early morning, daily and night-time symptoms, and early-morning limitation of activity in symptomatic patients compared with either tiotropium $18 \mu \mathrm{g}$ QD or placebo.

\begin{tabular}{|c|c|c|c|c|c|c|}
\hline \multirow[b]{2}{*}{$\begin{array}{l}\text { Change from baseline in normalised } \mathrm{FEV}_{1} \text { vs } \\
\text { placebo, } \mathrm{mL}\end{array}$} & \multicolumn{3}{|c|}{ Day 1} & \multicolumn{3}{|c|}{ Week 6} \\
\hline & $\begin{array}{l}\text { Aclidinium } \\
400 \mu g\end{array}$ & $\begin{array}{l}\text { Tiotropium } \\
18 \mu g\end{array}$ & $\begin{array}{l}\text { Aclidinium vs } \\
\text { tiotropium }\end{array}$ & $\begin{array}{l}\text { Aclidinium } \\
400 \mu \mathrm{g}\end{array}$ & $\begin{array}{l}\text { Tiotropium } \\
18 \mu \mathrm{g}\end{array}$ & $\begin{array}{l}\text { Aclidinium vs } \\
\text { tiotropium }\end{array}$ \\
\hline $\mathrm{FEV}_{1} \mathrm{AUC}_{-24 / 24 \mathrm{~h}}$ & $150^{*}$ & $87^{*}$ & $63 \dagger$ & $140^{* *}$ & $106^{*}$ & 34 \\
\hline $\mathrm{FEV}_{1} \mathrm{AUC}_{12-24 / 12 \mathrm{~h} \text { (night-time) }}$ & $157^{* *}$ & $67^{*}$ & $90 \dagger$ & $153^{* *}$ & $90^{* *}$ & $63 \dagger$ \\
\hline $\mathrm{FEV}_{1} \mathrm{AUC}_{-12}$ (day time) & $147^{* *}$ & $112^{* *}$ & 35 & $126^{*}$ & $123^{*}$ & 3 \\
\hline Morning pre-dose (trough) FEV ${ }_{1}$ & $136^{* *}$ & $68^{*}$ & $68 \dagger$ & $137^{*}$ & $70^{*}$ & $65 \dagger$ \\
\hline E-RS Total Score over 6 weeks & - & - & - & $-2.15^{*}$ & -0.98 & $-1.17 \dagger$ \\
\hline aEarly morning symptom severity over 6 weeks & - & - & - & $-0.25^{*}$ & -0.11 & $-0.14^{\dagger}$ \\
\hline (\% reduction) & & & & $(-9.54 \%)$ & $(-4.33 \%)$ & $(-5.21 \%)$ \\
\hline${ }^{\mathrm{b}}$ Night-time symptom severity over 6 weeks & - & - & - & $-0.23^{*}$ & -0.09 & $-0.14^{\dagger}$ \\
\hline (\% reduction) & & & & $(-10.31 \%)$ & $(-4.23 \%)$ & $(-6.09 \%)$ \\
\hline
\end{tabular}

${ }^{*} p<0.05$ vs placebo; ** $p \leq 0.0001$ vs placebo; ${ }^{\dagger} p<0.05$ vs tiotropium.

a Least squares mean change from baseline in the severity of early morning symptoms over 6 weeks: $1=$ No symptoms, $2=$ Mild, $3=$ Moderate, $4=$ Severe, $5=$ Very severe

${ }^{\mathrm{b} C h a n g e}$ from baseline in the severity of night-time symptoms over 6 weeks: $1=$ No symptoms, $2=$ Mild, $3=$ Moderate, $4=$ Severe, $5=$ Very severe.

AUC, area under the curve; COPD, chronic obstructive pulmonary disease; E-RS, EXAcerbations of Chronic pulmonary disease Tool-Respiratory Symptoms; FEV 1 , forced expiratory volume in $1 \mathrm{~s}$. 